\title{
Pedagogías emergentes en ambientes virtuales de aprendizaje
}

Emerging pedagogies in virtual learning environments

\author{
Oscar-Yecid Aparicio-Gómez ${ }^{1}$ \\ $E d \& T I C$ \\ Olga-Lucía Ostos-Ortiz ${ }^{2}$ \\ Universidad Santo Tomás
}

Recibido: 15.11 .2020

Aceptado: 29.11.2020

\section{Resumen}

Las pedagogías emergentes hacen su aparición en el escenario de los ambientes virtuales de aprendizaje en tiempos de gran incertidumbre. Las pedagogías emergentes apoyan el proceso educativo de las personas principalmente por medio de la promoción del aprendizaje continuo con tecnología y recursos educativos abiertos ante de grandes volúmenes de información y en contextos de redes de comunicación. Las metodologías activas para el aprendizaje coadyuvan en la gran labor de educar a partir de iniciativas provenientes de diversos lugares del mundo; así, el design thinking para resolver problemas, el llamado teachback para aprender a través de la conversación, el flipped learning para el acompañamiento y la independencia, la gamification para aprender a través de juegos y las social media para el aprendizaje en red, se hacen cada vez más recurrentes en las prácticas pedagógicas. Por otra parte, la evaluación de

\footnotetext{
1 oaparicio@editic.net https://orcid.org/0000-0003-3535-6288

https://scholar.google.com/citations?user=kH00EsUAAAAJ\&hl=es

2 olgaostosortiz@gmail.com https://orcid.org/0000-0002-6477-9872

https://scholar.google.com/citations?user=yCBpLUsAAAAJ\&hl=es
}

Volumen 1. Número 1. Enero - Junio 2021 ISSN: 2745-0341 (En línea) 
los aprendizajes comienza a considerar cada vez más la relevancia de las analíticas de aprendizaje, el blockchain, así como las insignias para acreditar el aprendizaje, en un contexto de evaluación discreta y formativa. Finalmente, las consideraciones éticas en torno al uso y la apropiación de las TIC en educación se hacen cada vez más necesarias para establecer los nexos entre pedagogía y tecnología; de esta manera, el aprendizaje basado en el contexto y, por lo tanto, también situado en el contexto de las historias personales, otorgando especial prevalencia al aprendizaje desde las emociones y los valores, requiere de todos los actores del proceso educativo que se acompañe el proceso de los niños y jóvenes desde una adecuada construcción del conocimiento desde una correcta argumentación en torno a sus intereses y necesidades.

Palabras clave: Educación, Teleformación, Educación basada en las competencias, Educación permanente, Aprendizaje continuo, Educación continua, Ética.

\section{Abstract}

Emerging pedagogies make their appearance on the stage of virtual learning environments in times of great uncertainty. Emerging pedagogies support the educational process of people mainly by promoting continuous learning with technology and open educational resources in the face of large volumes of information and in the context of communication networks. Active learning methodologies contribute to the great work of educating based on initiatives from different parts of the world; Thus, design thinking to solve problems, the so-called teachback to learn through conversation, flipped learning for accompaniment and independence, gamification to learn through games and social media for online learning, are made increasingly recurrent in pedagogical practices. On the other hand, the evaluation of learning begins to increasingly consider the relevance of learning analytics, the blockchain, as well as badges to accredit learning, in a context of discrete and formative evaluation. Finally, ethical considerations around the use and appropriation of ICT in education are becoming increasingly necessary to establish the links between pedagogy and technology; In this way, learning based on the context and, therefore, also situated in the context of personal 
stories, giving special prevalence to learning from emotions and values, requires all the actors in the educational process to follow the process of children and young people from an adequate construction of knowledge from a correct argumentation around their interests and needs.

Keywords: Education, Tele-training, Competency-based education, Lifelong education, Continuous learning, Continuous education, Ethics.

\section{Introducción}

Estamos aprendiendo en tiempos de incertidumbre, donde todo cambia muy rápido, donde no es fácil adaptarse a un cambio. Y en este contexto parece importante resaltar que nos encontramos con este tipo de transiciones no sólo en el campo educativo sino que se hace cada vez más evidente en el campo social, económico y cultural (Aparicio Gómez, Ostos Ortiz, Cortés Gallego, \& Abadía García, 2019). Podríamos llegar a plantearnos, tal como lo han hecho ya algunos autores, que estamos en una evolución del aprendizaje en la educación; hemos pasado del aprendizaje 1.0 al aprendizaje 4.0, tal como ya ha pasado con la Industria, la Conectividad, la Internet y la Web (Demartini \& Benussi, 2017).

En el contexto de la educación 1.0 había una persona sabía y la otra "que no", y uno que depositaba la información. En la educación 2.0, el profesor pasa a ser consejero, guía e intermediario. Por su parte, la educación 3.0 permite reconocer al profesor como quien lidera una creación compartida, cooperativa y colaborativa del conocimiento. Finalmente, en la educación 4.0 se hace una apuesta donde profesor emerge desde su figura mediadora, y con la disposición suficiente para interactuar con asistencias pedagógicas basadas en la inteligencia artificial (Brailas, Koskinas, \& Alexias, 2017).

Con respecto al acceso a los contenidos, en la educación 1.0 existe un soporte tradicional a derechos de autor accediendo al conocimiento mediante el pago de materiales o a través de las bibliotecas públicas o de la universidad. Con la educación 2.0 los recursos educativos pasan a ser abiertos y de acceso gratuito desde el acceso institucional. En la educación 3.0 se

Volumen 1. Número 1. Enero - Junio 2021 
accede a recursos educativos desde el entorno próximo del estudiante, y pueden ser creados y utilizados por los estudiantes, no solo se consume, sino que se produce conocimiento para ser puesto a disposición de todos. En la educación 4.0 se tiene acceso a grandes portales de aprendizaje basados en inteligencia artificial que integran los recursos educativos abiertos con los intereses de aprendizaje del estudiante (Pierucci, 2019).

En esta evolución, el estudiante ha dejado de ser un agente pasivo según el formato solicitado en la educación 1.0, e inicia la transición hacia la educación 2.0, donde comienza a tener un perfil activo, y se va configurando en el centro del proceso educativo; en la educación 3.0 el estudiante se siente acompañado por el profesor para ver qué necesidades y qué intereses tiene el estudiante, para hacer juntos el plan educativo. Finalmente, en la educación 4.0 el estudiante, desde el aprendizaje autónomo, pero con acompañamiento del profesor, apoyado por las nuevas tecnologías, por ejemplo, la inteligencia artificial (Tinmaz \& Lee, 2019).

\section{Pedagogías emergentes}

En las últimas décadas se ha hecho evidente que la relación entre pedagogía y tecnología, además de compleja, es simbiótica. Solo basta con mirar hacia atrás, a través de la historia, para constatar que el uso de la tecnología en el contexto de los aprendizajes, según la época, no ha evolucionado significativamente. Desde hace más de tres milenios se han usado tecnologías que aún se usan desde tiempos de la Academia de Platón y el Liceo de Aristóteles, constatado una escasa evolución de la tecnología educativa, manifiesta hasta los últimos haces en el uso de pizarras, libros de texto, enciclopedias y cuadernos. Existe una "pedagogía emergente" que está surgiendo en las últimas décadas en dialogo con las TIC de última generación y, que hunde sus raíces en ideas de grandes pedagogos del siglo XX, pero que va más en las prácticas innovadoras que realizan docentes intuitivos. Las pedagogías emergen como un conjunto de enfoques e ideas pedagógicas, que surgen alrededor del uso de las TIC en educación; por su parte, las tecnologías emergentes se identifican más con conceptos, herramientas e innovaciones utilizados en diversos contextos educativos (Cook \& Gregory, 2018; Moni, Mahmud, High, \& Carbajales-Dale, 2020; Neira, Salinas, \& Crosetti, 2017).

Volumen 1. Número 1. Enero - Junio 2021 
Aprender con tecnología se ha convertido en el gran referente para el uso de las TIC en la educación, apostando por su uso como herramientas cognitivas. La relación entre pedagogía y tecnología se puede reconocer en la ascendencia que hay ente una y otra; podemos acercarnos a las TIC como profesores "para aprender de las TIC"; o para aprender a usarlas en la vida diaria y reforzar las actitudes y valores "aprendiendo para las TIC"; o para acceder a los contenidos que proporcionan y aprender sobre las TIC; o finalmente, para aprender con las TIC como herramientas cognitivas (Greener, 2018; Sumuer, 2018; Trust, 2017; Wang \& Wegerif, 2019).

El aprendizaje con recursos educativos abiertos permite vincular a los estudiantes con el uso de la tecnología. Estos recursos están disponibles gratuitamente en línea para que todos puedan usarlos; y forman parte de ellos los contenidos y cursos abiertos, el material abierto para el desarrollo de capacidades de aprendizaje del personal docente, las herramientas de software libre, los depósitos de objetos de aprendizaje, así como todos los demás cursos educativos gratuitos. Los recursos educativos abiertos promueven oportunidades de aprendizaje permanente y fomentan el pensamiento crítico, creativo e independiente, a través del proceso de aprendizaje centrado en el estudiante (Chiappe \& Lee, 2017; Kim, Lee, Leite, \& Huggins-Manley, 2020; Rodríguez, Dodero, \& Alonso, 2011; Zhang \& Li, 2017).

Las nuevas formas de generación, distribución y acceso a los datos, su visualización y la interacción humana, hacen que estos cambien rápidamente. Por lo tanto, en la era del big data las personas no pueden ser simplemente receptores pasivos de informes basados en datos, su acción debe enfocarse en la exploración de datos, con el fin de administrar, adquirir, analizar, inferir y planificar, a partir de los datos. En este contexto, existe una importante demanda de alfabetización de datos en todas las disciplinas y áreas del conocimiento; por lo tanto, los estudiantes de hoy necesitan aprender a trabajar y pensar con datos desde una edad temprana, y así estar preparados para una sociedad basada en datos como en la que viven (Korkmaz \& Correia, 2019; Liang \& Hainan, 2019; Xu \& Ruan, 2018; Yuan, 2019). 
El aprendizaje continuo conecta las experiencias de aprendizaje en los contextos de tiempo, ubicación, uso de dispositivos y entornos sociales. En el aprendizaje continuo se conectan los aprendizajes desde casa con la institución educativa también a través de dispositivos y artefactos móviles; esta pedagogía forma parte de un proceso complejo, done los estudiantes pueden iniciar con una investigación e clase, recopilar datos en casa o en sus entornos habituales al aire libre, procesar la información haciendo uso de los recursos tecnológicos y luego compartir, hacer difusión y generar visibilidad de sus hallazgos en entornos sociales de aprendizaje tales como las aulas presenciales o virtuales (Barrett, Liu, \& Wang, 2020; Dilger, Gommers, \& Rapp, 2019; Hamid, Setyosari, Kuswandi, \& Ulfa, 2019).

El aprendizaje en red promueve entre conexiones entre los estudiantes, entre estudiantes y tutores, entre la comunidad de aprendizaje y sus propios recursos, así como entre los recursos que las diversas comunidades tienen a su disposición. Y aunque este tipo de conexiones pueden ser digitales, es innegable reconocer que las redes humanas que no siempre tienen acceso a internet para compartir información, también pueden compartir los recursos que poseen a través del uso de infraestructuras de servidores locales, por ejemplo, a través de un computador que pueda hacer sus veces en una sala y permita conectar a los participantes sin acceso a internet. El aprendizaje sin conexión en red une a las personas en actividades de cooperación generando espacios de aprendizaje más lecto, regulado y deliberado a la vez en las comunidades de aprendizaje sin conexión a internet (Bagheri \& Yamani douzi sorkhabi, 2020; L. H. J. Lee, Rahmat, Lin, Lim, \& Tan, 2020; Lötter \& Jacobs, 2020).

\section{Metodologías activas para el aprendizaje}

La llegada de las metodologías activas a los entornos físicos y virtuales de aprendizaje suponen cambios importantes en los enfoques de aprendizaje, donde resalta especialmente que debe estar centrado en el estudiante desde la autorregulación. Estas metodologías, entendidas como el conjunto de técnicas, estrategias y métodos para fomentar la participación activa el estudiante en su propio proceso de aprendizaje, pueden generar aprendizajes más significativos y profundos, orientar la adquisición de competencias, y facilitar su transferencia

Volumen 1. Número 1. Enero - Junio 2021 
a otros contextos y ámbitos de aprendizaje (Barroso-Osuna, Gutiérrez-Castillo, LlorenteCejudo, \& Ortiz, 2019; Herodotou et al., 2019; Parra-González, Belmonte, Segura-Robles, \& Cabrera, 2020). Entre muchas propuestas metodológicas, pueden desatacarse: Design thinking para resolver problemas, Teachback para aprender a través de la conversación, Flipped learning para el acompañamiento y la independencia, Gamification para aprender a través de juegos y Social media para el aprendizaje en red.

El Design thinking involucra una serie de episodios que generan nuevos escenarios de aprendizaje desde la creatividad y el pensamiento crítico; las competencias de análisis y construcción que pueden alcanzarse en su aplicación permiten resolver problemas a los estudiantes, teniendo en cuenta el la disponibilidad de materiales, así como las restricciones en su acceso. Las facilidades pedagógicas que supone enfrenar a los estudiantes a nuevos contextos que les permitan pensar en su mundo cotidiano y trabajar siguiendo una serie de pautas desde el pensamiento del diseño, permite a los estudiantes una compresión clara y distinta, fomentando la innovación y la resolución de problemas reales en las comunidades a donde pertenecen (Artiles \& Lande, 2016; Kumar, Zindani, \& Davim, 2020; Withell \& Haigh, 2018).

La forma de entender un tema y verificar que se ha entendido se puede llevar a cabo a través de una conversación estructurada. Este tipo de aprendizaje vinculado directamente a la retroalimentación consta de tres partes: En primer lugar, un experto explica un tema a alguien que es nuevo en este asunto; a continuación, el novato trata de explicar su nuevo entendimiento al experto; finalmente, si el novato coincide con el experto, éste continúa explicando un poco más sobre el tema. Si por el contrario, el novato lo explica con dificultad, el experto se tomará el tiempo necesario para aclarar la explicación y el novato dará la retroalimentación correspondiente hasta que pueda apropiarse de este conocimiento (Morrison \& Knoche, 2014; Tarimo \& Hickey, 2016, 2017).

El aprendizaje invertido revierte el enfoque tradicional de aprendizaje en el aula, y lo proyecta hacia los entonos habituales de interacción humana del estudiante. El Flipped learning, 
comprendido para el acompañamiento y la independencia, permite que el estudiante pueda aprender a su propio ritmo y desde sus propias necesidades e intereses en sus escenarios habituales, para luego socializarlo con sus pares y maestros. En el contexto digital, este tipo de metodología ofrece las posibilidades para que el proceso de aprendizaje se enmarque en un escenario donde los recursos de aprendizaje estén disponibles para los estudiantes y el profesor pueda orientarlo al aprendizaje independiente, mientras le apoya para desarrollar habilidades de gestión del tiempo, establecer espacios de aprendizaje en su entorno familiar y pueda probar diversas formas de trabajar a distancia (Gnutova, 2020; Kurbanoĝlu \& Akkoyunlu, 2017; Xiu \& Thompson, 2020).

Los juegos ahora son parte de la vida cotidiana de muchos niños y jóvenes, y en este contexto surge la gamification con el objetivo de aprender a través de juegos. Los buenos juegos suelen ser largos, complejos y difíciles, pero los jugadores persisten, y en cuanto mayor complejidad representa para el jugador, se motiva más a alcanzar los retos siguientes. El juego dispone a la resolución de problemas, mientras se recibe satisfacción y recompensas como retroalimentación. Por lo tanto, el juego se considera atractivo y motivador, donde se puede reconocer el equilibrio entre el desafío y su nivel de habilidad, una formulación de objetivos y recepción de retroalimentación clara, altos niveles de concentración, y una gran sensación gratificante. Cuando los juegos se usan en educación se promueve la creatividad y el desarrollo de habilidades como la colaboración y la resolución de problemas (Limantara, Meyliana, Hidayanto, \& Prabowo, 2019; Mahmud, Husnin, \& Soh, 2020; Panagiotarou, Stamatiou, Pierrakeas, \& Kameas, 2020; Su \& Cheng, 2015).

Finalmente, en un contexto mediado por el uso habitual y generalizado de las redes sociales para buscar amigos, compartir fotos, comprar bienes, escribir blogs, enviar mensajes, hacer videoconferencias, leer noticias, y pedir domicilios, entre muchas otras opciones, la social media representa en un escenario para el aprendizaje en red. Las redes sociales dan vida al aprendizaje al convocar diferentes personas y motivaciones en tiempos, espacios y posibilidades casi infinitas; este tipo de interacciones pueden apoyar la creatividad, la cooperación, la comunicación y el intercambio de información y recursos (Aldahdouh, 
Nokelainen, \& Korhonen, 2020; Höttecke \& Allchin, 2020; Liu et al., 2020; Matassi \& Boczkowski, 2020).

\section{Evaluación de los aprendizajes}

La participación de los estudiantes en los procesos de evaluación requiere diseñar la evaluación en coherencia con los resultados de aprendizaje y coherencia con la metodología propuesta, además de mantener vinculados los contenidos y planificar debidamente las etapas correspondientes; igualmente, debe ser de carácter continuado, viable, sostenible, participativo y estar centrado en la persona (Fernández-Ferrer \& Cano, 2019; Fernandez, Ion, \& Cano, 2014; Ion, Cano-García, \& Fernández-Ferrer, 2017; Serrat, Cano, \& Rubio, 2011). En este sentido, debe estar enfocada en la evaluación de un proceso de aprendizaje, más no de la valía de una persona; finalmente, el acompañamiento de la retroalimentación no está anclado en las actividades realizadas como en los procesos de aprendizaje futuros (Pons Seguí, Cano García \& Forés Miravalles, A., 2020).

La irrupción de las tecnologías digitales en los ambientes de aprendizaje puede considerarse como un soporte en los procesos de evaluación; en este horizonte, se requiere establecer los vínculos entre el diseño de aprendizaje y el análisis del aprendizaje. Este escenario permite comprender mejor cómo progresan los estudiantes, previo conocimiento de sus resultados, así como los objetivos de aprendizaje. La analítica de datos permite recopilar, analizar y presentar informes de grandes volúmenes de datos relacionados con los estudiantes y sus contextos de aprendizaje; estos análisis facilitan la retroalimentación del proceso evaluativo expresados en términos de recomendaciones diseñadas para acompañar la ruta de aprendizaje correspondiente a cada estudiante (Avila, Baldiris, Fabregat, \& Graf, 2020; Harindranathan \& Folkestad, 2019; Saqr \& Alamro, 2019; Shukor \& Abdullah, 2019).

Los datos resultantes deben ser almacenados de manera confiable mediante el uso, por ejemplo, de cadenas de bloques (Blockchain) que permita a las personas acceder a los resultados de sus propios procesos de aprendizaje, en términos de trabajos, ideas creativas, 
artículos, y certificados, entre otros. Hoy en día estas cadenas de bloques pueden garantizar el acceso, seguridad y distribución de los elementos que el usuario decida poner a disposición de la comunidad académica y profesional. Este tipo de sistemas basados en la confianza digital y la reputación académica de sus miembros facilita la distribución de la información de las personas en ambientes confiables y el acceso a sus perfiles en escenarios de reconocimiento académico y vinculaciones profesionales (Jirgensons \& Kapenieks, 2018; Li \& Wu, 2019).

En los procesos actuales de evaluación parece importante resaltar la preponderancia de una evaluación discreta, ya que ofrece la oportunidad de efectuar ajustes continuos en un entorno simulado (Aparicio-Gómez, 2016), por ejemplo, en entornos de software de modelado o videojuegos, teniendo en cuenta cada uno de los estudiantes en función de sus necesidades, intereses y de los objetivos de aprendizaje. En estas condiciones, es muy probable que el estudiante no advierta que el aprendizaje y la evaluación forman parte del juego. Este tipo de evaluación discreta facilita la detección de habilidades en la resolución de problemas que implican ciertos niveles de creatividad, innovación y pensamiento crítico (Georgiadis, van Lankveld, Bahreini, \& Westera, 2019; Georgiadis, Van Lankveld, Bahreini, \& Westera, 2019; Min et al., 2019).

Actualmente, se detecta habitualmente que los estudiantes buscan la manera de monitorear su propio desempeño, por lo que la retroalimentación en los procesos de evaluación de los aprendizajes deben basarse en procesos de autogestión; de esta manera, la evaluación formativa debe enfocarse hacia los beneficios inmediatos que puede obtener el estudiante en la medida que los profesores puedan orientarle sobre cómo reducir la brecha entre el desempeño actual y el desempeño deseado, teniendo en cuenta sus intereses y necesidades, así como los objetivos de aprendizaje. Se requiere, por tanto, aclarar lo que se considera como buen desempeño, facilitar la autoevaluación, fomentar el diálogo con pares y profesores, así como la autoestima; y utilizar la retroalimentación para mejorar el aprendizaje (Georgiadis, van Lankveld, et al., 2019; Georgiadis, Van Lankveld, et al., 2019; Min et al., 2019). 
Finalmente, parece importante considerar que los estudiantes requieren una serie de estímulos que trascienden la retroalimentación y las orientaciones de sus maestros, permitiendo la emergencia de diversos tipos de estímulos que puedan adecuarse a los diversos entornos de presencialidad y virtualidad. En este escenario aparecen las insignias digitales, y las mochilas donde estas son almacenadas, como una herramienta para acreditar el aprendizaje, a la vez que motivan la autoestima, la cooperación, y el reconocimiento de los logros del estudiante como el camino hacia la consecución de objetivos más sólidos (Beattie, 2016; McDaniel, 2016; Ostashewski \& Reid, 2015).

\section{4. Ética, educación y tecnología}

La ética como reflexión profunda sobre la moral, sobre lo que habitualmente hacemos, pero reconociendo que se hace con una intencionalidad muy clara, con la idea de perfeccionar aquello que ya tenemos entre manos. En este ejercicio de pensar, de debatir, de ser críticos, desde una crítica argumentada, surge la necesidad de plantearse el uso y la apropiación de las TIC como herramientas cognitivas en los procesos de aprendizaje (Griffiths, 2020; Overstreet, 2016; Tracy \& Carmichael, 2013).

La palabra ética originalmente significa "madriguera", el entorno toma las características de la persona, su forma, su aroma, su manera de moverse, su manera de ser. Se trata de instalarse en un contexto moral, pero impregnándolo de su propia identidad. El aprendizaje basado en el contexto reclama esta máxima ética de instalarse, en aquello que le rodea, pero a la vez en aquello que se crea. El contexto es como se da sentido a la experiencia, entre lo relevante y lo irrelevante. El aprendizaje basado en el contexto permite sentirse situado y comprendido, respetado y motivado a transformar el entorno, a mejorarlo (Dori, Avargil, Kohen, \& Saar, 2018; Nijenhuis-Voogt, Meijer, \& Barendsen, 2018; Wen \& Katt, 2019).

En esta amplia variedad de contextos, de horizontes de referencia, se reconoce una importancia especial a las emociones que surgen de la realidad propia de cada persona; sus diversas maneras de ser, necesidades e intereses sitúan el foco de la reflexión pedagógica en

Volumen 1. Número 1. Enero - Junio 2021 
la relevancia de identificar las emociones para orientar el proceso educativo de cada persona. Además de la importancia de efectuar este tipo de reconocimientos de persona a persona, ya en el contexto de los entornos virtuales surgen enfoques avanzados de inteligencia artificial para identificar, reconocer y acompañar el proceso de aprendizaje de cada estudiantes teniendo en cuenta sus emociones a lo largo de todo el proceso (Ma, Sun, Song, Li, \& Xu, 2018; O’Reilly, 2015; Yang, Alsadoon, Prasad, Singh, \& Elchouemi, 2018). Surgen también desafíos éticos para responder correctamente a este tipo de nuevos reconocimientos y acompañamientos mediados por tecnologías digitales.

El aprendizaje se hace cada vez más ubicuo y más deslocalizado, ya no se aprende para solo para el presente; las competencias que requiere un universo en permanente transformación requieren de un aprendizaje permanente para apropiarse del futuro, para reaccionar rápida y asertivamente ante estos cambios acelerados con resiliencia y determinación. La misión de preparar a los estudiantes ante un futuro impredecible, un mundo incierto y un entorno laboral y de continuo aprendizaje requiere, por parte de los maestros, conectar el aprendizaje con los problemas del mundo real (Fadeeva, Galkute, Mader, \& Scott, 2014; W. O. Lee, 2012; Winter, Cotton, \& Warwick, 2016). Se requieren habilidades para aprender y volver a aprender permanentemente, disposiciones que permitan cambiar de perspectiva teniendo en cuenta las nuevas informaciones que surgen, en términos de habilidades cognitivas y de comprensiones culturales e interpersonales.

Los horizontes de referencia que proceden especialmente de las familias de origen deben ser considerados en los procesos de aprendizaje que reconocen y promueven los valores internos. La libertad de elegir desde una conciencia formada, desde una toma de posesión del propio aprendizaje, crea el ambiente idóneo para que florezca el aprendizaje significativo a lo largo de toda la vida, aquel aprendizaje que nos permite activar, controlar y apropiarnos de nuestra propia historia (Ben-Horin et al., n.d.; Sagy, Kali, Tsaushu, \& Tal, 2018; Sánchez-Maroño et al., 2014). Los maestros, por su parte, tienen la responsabilidad de coadyuvar, orientar y acompañar a los estudiantes en sus procesos de apropiación y el equilibrio de sus aprendizajes entre sus valores internos y los valores normativos propios de la sociedad. 
Con el fin de alcanzar el aprendizaje autónomo, pero autogestionado con un importante componente de heteronomía, se requiere consolidar el aprendizaje a través de la argumentación. Este enfoque prepara a los estudiantes para profundizar en fuentes de información validadas por pares y construir un discurso disciplinar y transdisciplinar desde la construcción de un discurso lógico, racional, crítico y argumentativo en torno a la evidencia de la ciencia. Los estudiantes podrán comprender mejor las ideas científicas, sus procesos y resultados de investigación, mientras se apropian del lenguaje con claridad, distinción y precisión (Asterhan \& Babichenko, 2015; Berland \& Russ, 2017; Reznitskaya \& Švaříček, 2019).

\section{Conclusiones}

Las pedagogías emergentes en ambientes virtuales de aprendizaje se enmarcan en los procesos de innovación educativa y gestión del conocimiento (Ostos-Ortiz, \& González Gil, 2020). En este sentido la agrupación que se ha hecho en cuatro grandes temas, a saber: pedagogías emergentes, metodologías activas para el aprendizaje, evaluación de los aprendizajes, y ética, educación y tecnología, ofrecen al lector una visión de conjunto para profundizar en cada uno de los puntos de reflexión allí consignados.

Las pedagogías emergentes que surgen en diálogo con las tecnologías emergentes, hunden sus raíces en las propuestas de los grandes pedagogos del siglo XX; sin embargo, su grandeza está en el proceso de reflexión y apropiación por parte de maestros intuitivos para construir propuestas de innovación educativa a través de las prácticas innovadoras que responden al lenguaje de los estudiantes y de la sociedad actual.

En este sentido, las metodologías activas para el aprendizaje, refuerzan el cambio de enfoque a través de una serie de iniciativas que generan aprendizajes significativos y profundos, a la vez que permiten alcanzar competencias en términos de capacidad de análisis y síntesis, razonamiento crítico, conexión entre la teoría y la práctica, resolución de problemas, 
capacidad comunicativa y social mediante la expresión oral y escrita; así como liderazgo, trabajo cooperativo y compromiso ético.

La evaluación de los aprendizajes reviste un interés especial en términos de elegir una opción sólida por evaluar el potencial de los estudiantes para aprender en lugar de medir lo que han conseguido. La dinámica de la evaluación nos pone en un escenario prospectivo, de evaluación dinámica hacia el futuro, teniendo en cuenta lo que se ha aprendido, pero como referente para las nuevas maneras de aprender y de aprehender lo aprendido para construir nuevos entornos de aprendizaje a lo largo de toda la vida.

Finalmente, los aprendizajes requieren hundir sus raíces en las fuentes de la ética y su relación con educación en entornos digitales. El conocimiento profundo sobre los referentes universales para la valoración de situaciones que se presentan en los procesos de aprendizaje, desde unos horizontes de referencia consensuados socialmente, tales como los valores, permitirán que nuevos referentes puedan emerger a medida que emergen nuevas situaciones en los contextos de aprendizaje a lo largo de toda la vida.

\section{Referencias}

Aldahdouh, T. Z., Nokelainen, P., \& Korhonen, V. (2020). Technology and Social Media Usage in Higher Education: The Influence of Individual Innovativeness. SAGE Open, 10(1). https://doi.org/10.1177/2158244019899441

Aparicio-Gómez, Jaime-Orlando (2016). El diseño tecnopedagógico en la educación básica primaria, secundaria y media. En Aparicio-Gómez, Oscar-Yecid (Eds.). El Uso Educativo de las TIC (pp. 11-23). Bogotá, Colombia: Universidad Central. ISBN: 978-958-26-0316-8

Artiles, J. A., \& Lande, M. (2016). Broadening non-designers' solutions for big issues: The Education DesignShop design thinking workshop. International Journal of Engineering Education, 32(3), 1418-1427. Tempus Publications. 
Asterhan, C. S. C., \& Babichenko, M. (2015). The social dimension of learning through argumentation: Effects of human presence and discourse style. Journal of Educational Psychology, 107(3), 740-755. https://doi.org/10.1037/edu0000014

Avila, C., Baldiris, S., Fabregat, R., \& Graf, S. (2020). Evaluation of a learning analytics tool for supporting teachers in the creation and evaluation of accessible and quality open educational resources. British Journal of Educational Technology, 51(4), 1019-1038. https://doi.org/10.1111/bjet.12940

Bagheri, A., \& Yamani douzi sorkhabi, M. (2020). Looking for value through networked learning in social context. Education and Training. https://doi.org/10.1108/ET-10-2019-0223

Barrett, N. E., Liu, G. Z., \& Wang, H. C. (2020). Seamless learning for oral presentations: designing for performance needs. Computer Assisted Language Learning. https://doi.org/10.1080/09588221.2020.1720254

Barroso-Osuna, J., Gutiérrez-Castillo, J. J., Llorente-Cejudo, M. C., \& Ortiz, R. V. (2019). Difficulties in the incorporation of augmented reality in university education: Visions from the experts. Journal of New Approaches in Educational Research, 8(2), 126-141. https://doi.org/10.7821/naer.2019.7.409

Beattie, S. (2016). Height vs. Depth in badging framework design. In Foundation of Digital.

Badges and Micro-Credentials: Demonstrating and Recognizing Knowledge and Competencies (pp. 307-324). https://doi.org/10.1007/978-3-319-15425-1_17

Ben-Horin, H., Pion, C., Kali, Y., Eshet-Alkalai, Y., Blau, I., Caspi, A., ... Silber-Varod, V. (n.d.). E 1 Designing Technology to Foster Socioscientific Reasoning by Promoting Internal Values of Learning. 
Berland, L. K., \& Russ, R. S. (2017). Conceptual change through argumentation: A process of dynamic refinement. In Converging Perspectives on Conceptual Change: Mapping an Emerging Paradigm in the Learning Sciences (pp. 180-189). https://doi.org/10.4324/9781315467139

Brailas, A., Koskinas, K., \& Alexias, G. (2017). Teaching to emerge: Toward a bottom-up pedagogy. Cogent Education, 4(1). https://doi.org/10.1080/2331186X.2017.1377506

Chiappe, A., \& Lee, L. L. (2017). Open teaching: A new way on e-learning? Electronic Journal of E-Learning, 15(5), 369-383.

Cook, V. S., \& Gregory, R. L. (2018). Emerging technologies: It's not what you say - It's what they do. Online Learning Journal, 22(3), 121-130. https://doi.org/10.24059/olj.v22i3.1463

Demartini, C., y Benussi, L. (2017). Do Web 4.0 and Industry 4.0 Imply Education X.0? IT Pro May/June 2017, IEEE Computer Society.

Dilger, B., Gommers, L., \& Rapp, C. (2019). The learning problems behind the seams in seamless learning. In Lecture Notes in Educational Technology (pp. 29-51). https://doi.org/10.1007/978-981-13-3071-1_2

Dori, Y. J., Avargil, S., Kohen, Z., \& Saar, L. (2018). Context-based learning and metacognitive prompts for enhancing scientific text comprehension. International Journal of Science Education, 40(10), 1198-1220. https://doi.org/10.1080/09500693.2018.1470351

Fadeeva, Z., Galkute, L., Mader, C., \& Scott, G. (2014). Assessment for transformation higher education thrives in redefining quality systems. In Palgrave Studies in Global Higher Education (pp. 1-22). https://doi.org/10.1057/9781137459145_1 
Fernandez, M., Ion, G., \& Cano, E. (2014). Educating for competencies development in higher education with technology. Proceedings of the European Conference on E-Learning, ECEL, 2014-January, 704-707. Academic Conferences Limited.

Fernández-Ferrer, M., \& Cano, E. (2019). Feedback experiences to improve the continuous assessment: The use of Twitter as an emerging technology. Educar, 55(2), 437-455. https://doi.org/10.5565/rev/educar.872

Georgiadis, K., van Lankveld, G., Bahreini, K., \& Westera, W. (2019). Reinforcing stealth assessment in serious games. Lecture Notes in Computer Science (Including Subseries Lecture Notes in Artificial Intelligence and Lecture Notes in Bioinformatics), 11899 LNCS, 512-521. https://doi.org/10.1007/978-3-030-34350-7_49

Georgiadis, K., Van Lankveld, G., Bahreini, K., \& Westera, W. (2019). Learning analytics should analyse the learning: Proposing a generic stealth assessment tool. IEEE Conference on Computatonal Intelligence and Games, CIG, 2019-August. https://doi.org/10.1109/CIG.2019.8847960

Gnutova, I. I. (2020). From flipped classroom to flipped learning: Evolution of the concept and its philosophical foundations. Vysshee Obrazovanie v Rossii, 29(3), 86-95. https://doi.org/10.31992/0869-3617-2020-29-3-86-95

Greener, S. (2018, October 3). The knowing-doing gap in learning with technology. Interactive Learning Environments, Vol. 26, pp. 856-857. https://doi.org/10.1080/10494820.2018.1510155

Griffiths, D. (2020). The Ethical Issues of Learning Analytics in Their Historical Context. In Lecture Notes in Educational Technology (pp. 39-55). https://doi.org/10.1007/978-981-154276-3_3 
Hamid, A., Setyosari, P., Kuswandi, D., \& Ulfa, S. (2019). The implementation of mobile seamless learning strategy in mastering students' concepts for elementary school. Journal for the Education of Gifted Young Scientists, 7(4), 967-982. https://doi.org/10.17478/jegys.622416

Harindranathan, P., \& Folkestad, J. (2019). Learning analytics to inform the learning design: Supporting instructor's inquiry into student learning in unsupervised technology-enhanced platforms. Online Learning Journal, 23(3), 34-55. https://doi.org/10.24059/olj.v23i3.2057

Herodotou, C., Sharples, M., Gaved, M., Kukulska-Hulme, A., Rienties, B., Scanlon, E., \& Whitelock, D. (2019, October 11). Innovative Pedagogies of the Future: An Evidence-Based Selection. Frontiers in Education, Vol. 4. https://doi.org/10.3389/feduc.2019.00113

Höttecke, D., \& Allchin, D. (2020). Reconceptualizing nature-of-science education in the age of social media. Science Education. https://doi.org/10.1002/sce.21575

Ion, G., Cano-García, E., \& Fernández-Ferrer, M. (2017). Enhancing self-regulated learning through using written feedback in higher education. International Journal of Educational Research, 85, 1-10. https://doi.org/10.1016/j.ijer.2017.06.002

Jirgensons, M., \& Kapenieks, J. (2018). Blockchain and the Future of Digital Learning Credential Assessment and Management. Journal of Teacher Education for Sustainability, 20(1), 145-156. https://doi.org/10.2478/jtes-2018-0009

Kim, D., Lee, Y., Leite, W. L., \& Huggins-Manley, A. C. (2020). Exploring student and teacher usage patterns associated with student attrition in an open educational resourcesupported online learning platform. Computers and Education, 156. https://doi.org/10.1016/j.compedu.2020.103961 
Korkmaz, C., \& Correia, A. P. (2019). A review of research on machine learning in educational technology. Educational Media International, 56(3), 250-267. https://doi.org/10.1080/09523987.2019.1669875

Kumar, K., Zindani, D., \& Davim, J. P. (2020). Digital Thinking Integrated with Design Thinking. In SpringerBriefs in Applied Sciences and Technology (pp. 69-74). https://doi.org/10.1007/978-3-030-31359-3_6

Kurbanoĝlu, S., \& Akkoyunlu, B. (2017). Information Literacy and Flipped Learning. In Pathways into Information Literacy and Communities of Practice: Teaching Approaches and Case Studies (pp. 53-84). https://doi.org/10.1016/B978-0-08-100673-3.00003-4

Lee, L. H. J., Rahmat, R. B., Lin, L., Lim, P. H., \& Tan, T. H. (2020). The development of an implementation framework to support knowledge construction in online networked learning. Professional Development in Education. https://doi.org/10.1080/19415257.2020.1763430

Lee, W. O. (2012, February). Learning for the future: The emergence of lifelong learning and the internationalisation of education as the fourth way? Educational Research for Policy and Practice, Vol. 11, pp. 53-64. https://doi.org/10.1007/s10671-011-9122-9

Li, L., \& Wu, X. (2019). Research on school teaching platform based on blockchain technology. 14th International Conference on Computer Science and Education, ICCSE 2019, 38-43. https://doi.org/10.1109/ICCSE.2019.8845353

Liang, Q., \& Hainan, N. C. (2019). Adaptive Learning Model and Implementation Based on Big Data. 2019 2nd International Conference on Artificial Intelligence and Big Data, ICAIBD 2019, 183-186. https://doi.org/10.1109/ICAIBD.2019.8836984

Limantara, N., Meyliana, Hidayanto, A. N., \& Prabowo, H. (2019, February 1). The elements of gamification learning in higher education: A systematic literature review. International 
Journal of Mechanical Engineering and Technology, Vol. 10, pp. 982-991. IAEME Publication.

Liu, Y., Luo, Q., Shen, H., Zhuang, S., Xu, C., Dong, Y., ... Deng, H. (2020). Social media big data-based research on the influencing factors of insomnia and spatiotemporal evolution. IEEE Access, 8, 41516-41529. https://doi.org/10.1109/ACCESS.2020.2976881

Lötter, M. J., \& Jacobs, L. (2020). Using smartphones as a social constructivist pedagogical tool for inquiry-supported problem-solving: an exploratory study. Journal of Teaching in Travel and Tourism. https://doi.org/10.1080/15313220.2020.1715323

Ma, C., Sun, C., Song, D., Li, X., \& Xu, H. (2018). A deep learning approach for online learning emotion recognition. 13th International Conference on Computer Science and Education, ICCSE 2018, 567-571. https://doi.org/10.1109/ICCSE.2018.8468741

Mahmud, S. N. D., Husnin, H., \& Soh, T. M. T. (2020). Teaching presence in online gamified education for sustainability learning. Sustainability (Switzerland), 12(9). https://doi.org/10.3390/su12093801

Matassi, O., \& Boczkowski, P. J. (2020). Social media in ibero-America. Review article. Profesional de La Informacion, Vol. 29. https://doi.org/10.3145/epi.2020.ene.04

McDaniel, R. (2016). What we can learn about digital badges from video games. In Foundation of Digital Badges and Micro-Credentials: Demonstrating and Recognizing Knowledge and Competencies (pp. 325-342). https://doi.org/10.1007/978-3-319-15425-1_18

Min, W., Frankosky, M., Mott, B. W., Rowe, J., Smith, P. A. M., Wiebe, E., ... Lester, J. (2019). DeepStealth: Game-Based Learning Stealth Assessment with Deep Neural Networks. IEEE Transactions on Learning Technologies. https://doi.org/10.1109/TLT.2019.2922356 
Moni, S. M., Mahmud, R., High, K., \& Carbajales-Dale, M. (2020). Life cycle assessment of emerging technologies: A review. Journal of Industrial Ecology, 24(1), 52-63. https://doi.org/10.1111/jiec.12965

Morrison, A., \& Knoche, H. (2014). Design transformations: Teaching design through evaluations. Kybernetes, 43, 1372-1380. https://doi.org/10.1108/K-07-2014-0153

Neira, E. A. S., Salinas, J., \& Crosetti, B. de B. (2017). Emerging Technologies (ETs) in education: A systematic review of the literature published between 2006 and 2016 . International Journal of Emerging Technologies in Learning, 12(5), 128-149. https://doi.org/10.3991/ijet.v12i05.6939

Nijenhuis-Voogt, J., Meijer, P. C., \& Barendsen, E. (2018). Context-based teaching and learning of fundamental computer science concepts: Exploring teachers' ideas. ACM International Conference Proceeding Series. https://doi.org/10.1145/3265757.3265772

O'Reilly, M. (2015). The influence of emotions, attitudes and perceptions on learning with technology. Research in Learning Technology, Vol. 23. https://doi.org/10.3402/rlt.v23.27763

Ostashewski, N., \& Reid, D. (2015). A history and frameworks of digital badges in education. In Gamification in Education and Business (pp. 187-200). https://doi.org/10.1007/978-3-31910208-5_10

Ostos-Ortiz, Olga-Lucía \& González Gil, E. (2020). Gestión del conocimiento, un reto en la educación superior. En: Aparicio-Gómez, Oscar-Yecid y Ostos-Ortiz, Olga-Lucía. (Eds.). Innovación Educativa y Gestión del Conocimiento (pp. 19-42). Bogotá, Colombia: Universidad Santo Tomás. ISBN: 978-958-782-304-2 
Overstreet, M. (2016). Principled/digital: Composition's "ethics of attunement" and the writing MOOC. In Handbook of Research on Writing and Composing in the Age of MOOCs (pp. 75-86). https://doi.org/10.4018/978-1-5225-1718-4.ch005

Panagiotarou, A., Stamatiou, Y. C., Pierrakeas, C., \& Kameas, A. (2020). Gamification acceptance for learners with different E-skills. International Journal of Learning, Teaching and Educational Research, 19(2), 263-278. https://doi.org/10.26803/IJLTER.19.2.16

Parra-González, M. E., Belmonte, J. L., Segura-Robles, A., \& Cabrera, A. F. (2020). Active and emerging methodologies for ubiquitous education: Potentials of flipped learning and gamification. Sustainability (Switzerland), 12(2). https://doi.org/10.3390/su12020602

Pierucci, L. (2019). Challenges for teaching wireless communications standards at the graduate level. Education Sciences, 9(4). https://doi.org/10.3390/educsci9040298

Pons Seguí, L., Cano García, E. \& Forés Miravalles, A. (2020). Fortalecimiento del juicio evaluativo con apoyo de la tecnología en la sociedad del conocimiento. En: Aparicio-Gómez, Oscar-Yecid y Ostos-Ortiz, Olga-Lucía. (Eds.). Innovación Educativa y Gestión del Conocimiento (pp. 101-134). Bogotá, Colombia: Universidad Santo Tomás. ISBN: 978-958782-304-2

Reznitskaya, A., \& Švaříček, R. (2019). Better learning through argumentation. Studia Paedagogica, Vol. 24, pp. 5-10. Masaryk University, Faculty of Arts.

Rodríguez, J. S., Dodero, J. M., \& Alonso, S. S. (2011). Determinando la relevancia de los recursos educativos abiertos a través de la integración de diferentes indicadores de calidad. Revista de Universidad y Sociedad Del Conocimiento, 8(2), 211-224. 
Sagy, O., Kali, Y., Tsaushu, M., \& Tal, T. (2018). The Culture of Learning Continuum: promoting internal values in higher education. Studies in Higher Education, 43(3), 416-436. https://doi.org/10.1080/03075079.2016.1174205

Sánchez-Maroño, N., Alonso-Betanzos, A., Fontenla-Romero, O., Brinquis-Núñez, C., Polhill, J. G., \& Craig, T. (2014). Influence of internal values and social networks for achieving sustainable organizations. Frontiers in Artificial Intelligence and Applications, 263, 1179-1184. https://doi.org/10.3233/978-1-61499-419-0-1179

Saqr, M., \& Alamro, A. (2019). The role of social network analysis as a learning analytics tool in online problem-based learning. BMC Medical Education, 19(1). https://doi.org/10.1186/s12909-019-1599-6

Serrat, N., Cano, E., \& Rubio, A. (2011). Learning self-regulation competences in higher education by using ICT. International Journal of Learning, 17(11), 1-16. https://doi.org/10.18848/1447-9494/cgp/v17i11/47347

Shukor, N. A., \& Abdullah, Z. (2019). Using learning analytics to improve MOOC instructional design. International Journal of Emerging Technologies in Learning, 14(24), 617. https://doi.org/10.3991/ijet.v14i24.12185

Su, C. H., \& Cheng, C. H. (2015). A mobile gamification learning system for improving the learning motivation and achievements. Journal of Computer Assisted Learning, 31(3), 268286. https://doi.org/10.1111/jcal.12088

Sumuer, E. (2018). Factors related to college students' self-directed learning with technology. Australasian Journal of Educational Technology, 34(4), 29-43. https://doi.org/10.14742/ajet.3142 
Tarimo, W. T., \& Hickey, T. J. (2016). Fully integrating remote students into a traditional classroom using live-streaming and TeachBack. Proceedings - Frontiers in Education Conference, FIE, 2016-November. https://doi.org/10.1109/FIE.2016.7757746

Tarimo, W. T., \& Hickey, T. J. (2017). Groupwork: Learning during collaborative assessment activities. Computer-Supported Collaborative Learning Conference, CSCL, 1, 463-470. International Society of the Learning Sciences (ISLS).

Tinmaz, H., \& Lee, J. H. (2019). A preliminary analysis on Korean University students' readiness level for industry 4.0 revolution. Participatory Educational Research, 6(1), 70-83. https://doi.org/10.17275/per.19.6.6.1

Tracy, F., \& Carmichael, P. (2013). Research ethics and participatory research in an interdisciplinary technology-enhanced learning project. In Ethics and Academic Freedom in Educational Research (pp. 41-53). https://doi.org/10.4324/9781315872711

Trust, T. (2017, April 3). Preparing Future Teachers to Redefine Learning With Technology. Journal of Digital Learning in Teacher Education, Vol. 33, pp. 44-45. https://doi.org/10.1080/21532974.2017.1281654

Wang, M., \& Wegerif, R. (2019). From active-in-behaviour to active-in-thinking in learning with technology. British Journal of Educational Technology, Vol. 50, pp. 2178-2180. https://doi.org/10.1111/bjet.12874

Wen, S. F., \& Katt, B. (2019). Toward a Context-Based Approach for Software Security Learning. Journal of Applied Security Research, 14(3), 288-307. https://doi.org/10.1080/19361610.2019.1585704 
Winter, J., Cotton, D., \& Warwick, P. (2016). The University as a Site of Socialisation for Sustainability Education. In World Sustainability Series (pp. 97-108). https://doi.org/10.1007/978-3-319-32928-4_7

Withell, A., \& Haigh, N. (2018). Enhancing curricula and learning environments using critical realist perspectives and approaches: a case study and critique. Curriculum Journal, 29(3), 319337. https://doi.org/10.1080/09585176.2017.1400451

Xiu, Y., \& Thompson, P. (2020). Flipped university class: A study of motivation and learning. Journal of Information Technology Education: Research, 19, 41-63. https://doi.org/10.28945/4500

Xu, N., \& Ruan, B. (2018). An Application of Big Data Learning Analysis Based on MOOC Platform. Proceedings - 9th International Conference on Information Technology in Medicine and Education, ITME 2018, 698-702. https://doi.org/10.1109/ITME.2018.00159

Yang, D., Alsadoon, A., Prasad, P. W. C., Singh, A. K., \& Elchouemi, A. (2018). An Emotion Recognition Model Based on Facial Recognition in Virtual Learning Environment. Procedia Computer Science, 125, 2-10. https://doi.org/10.1016/j.procs.2017.12.003

Yuan, L. (2019). Discussion on the application of computer big data in internet learning. Proceedings - 2019 12th International Conference on Intelligent Computation Technology and Automation, ICICTA 2019, 482-486. https://doi.org/10.1109/ICICTA49267.2019.00109

Zhang, M., \& Li, Y. (2017). Teaching experience on faculty members' perceptions about the attributes of open educational resources (OER). International Journal of Emerging Technologies in Learning, 12(4), 191-199. https://doi.org/10.3991/ijet.v12i04.6638 
REVISTA INTERNACIONAL DE PEDAGOGÍA E INNOVACIÓN EDUCATIVA |

Volumen 1. Número 1. Enero - Junio 2021 ISSN: 2745-0341 (En línea) 